\title{
Role of Ulipristal Acetate as a Treatment for Fibroid Uterus
}

\author{
Tahira Shahzadi* \\ Department of Obstetrics and Gynecology, Dr. Sulaiman Al Habib Hospital, Saudi Arabia
}

*Corresponding author: Tahira Shahzadi, Department of Obstetrics and Gynecology, Dr. Sulaiman Al Habib Hospital, Khurais

Road, Ar Rayan, Riyadh, Saudi Arabia

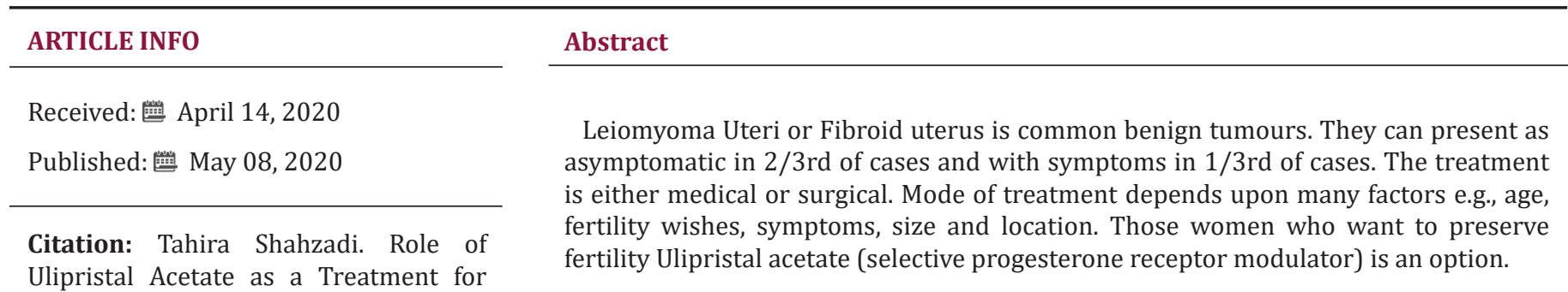

Fibroid Uterus. Biomed J Sci \& Tech Res

27(4)-2020. BJSTR. MS.ID.004520.

\section{Mode of Action}

SPRMs (selective progesterone receptor modulators) are synthetic compounds that act at Progesterone receptor sites showing either agonistic or antagonistic activity depending upon changes in progesterone receptors [1,2]. Progesterone has role in Fibroids cell proliferation. In studies, it has been shown that SPRMs inhibit fibroid cell proliferation by causing apoptosis, antifibrinolytic activity and reducing or blocking growth factor expression. That's why SPRMs may contribute to reducing fibroid size and reducing heavy blood loss. SPRMs can cause changes in endometrium known as progesterone receptor modulator associated endometrial changes (PAECs). These changes include endometrial gland cystic dilatation with irregular architecture lined with inactive glands and non decidualized stroma. So could not be classified as proliferative or secretary endometrium [1,2]. $\mathrm{GnRH}$ agonists cause down regulation at pituitary gland and have an affect on GnRH receptors resulting in decrease in serum estradiol and progesterone levels. In comparison SPRMs affects at three levels directly pituitary gland, fibroid, and endometrium [1]. It causes amenorrhea by having affect on pituitary gland, by inhibiting ovulation and at same time maintains estradiol level [3].
SPRMs also result in reducing size of fibroid by causing apoptosis [4].

\section{Role of SPRMs to Reduce Size of Fibroid Preoperatively and Long Term Medical Management}

So far four PEARL trials have been conducted. PEARL I was a randomised, double-blind, placebo controlled trial. In this trial evaluation of women was done with symptomatic fibroids with UPA $5 \mathrm{mg}$ or $10 \mathrm{mg}$ tablet for three months. After 3 months it was found that uterine bleeding improved in 91\% who were taking $5 \mathrm{mg}$ UPA and $92 \%$ who were taking $10 \mathrm{mg}$ UPA and only $19 \%$ in Placebo group. It was found that improvement of anaemia in treatment group which again have resulted in reduced morbidity and mortality in patient for surgery. PEARL II trial compared two group one received 5 or $10 \mathrm{mg}$ UPA while other received GnRH (leuprolide acetate depot) $3.75 \mathrm{mg}$ injection once a month. It was found that control of uterine bleeding in $90 \%$ receiving $5 \mathrm{mg}$ UPA and $98 \%$ receiving $10 \mathrm{mg}$ UPA and $89 \%$ received GnRH. The median time was 5-7 days receiving UPA either 5 or $10 \mathrm{mg}$ and 21 days receiving GnRH for control of bleeding. The effect of UPA was sustained up to six months after treatment $[5,2,6]$. PEARL III It was studied 
safety and effect of intermittent long term three month courses of $10 \mathrm{mg}$ UPA for treatment of fibroids. Efficacy was assessed in term of amenorrhea and median time for control of bleeding after one, two, three and four courses. No Hyperplasia was found on endometrial histopathology. So it was found that repeated courses of UPA can improve the benefits in term of control of bleeding and reducing fibroid size. PEARL IV trial they assessed improvement in pain, Quality of life and reduction in fibroid size after repeated 12 week courses of UPA 5 or $10 \mathrm{mg}$ daily for intermittent treatment. Pregnancies and outcome have been reported after treatment with UPA $[5,2,7]$.

\section{Efficacy of Intermittent Long Term Repeated Three Month Courses of UPA 10 mg}

The effect of intermittent long term UPA $10 \mathrm{mg}$ in repeated courses of three month with a gap of two menstrual cycles between courses was assessed and it was found that improvement in terms of bleeding control, reduced time to achieve amenorrhea. Fibroid size was reduced by more than $50 \%$ with each subsequent course. So treatment of fibroid depends on many factors like age, future fertility wishes, symptoms and location of fibroids [1,3-5]. If age is less than 40 yrs with uterine cavity deformed by fibroids and infertility, then according to International Federation of Gynaecology and Obstetrics classification type 0 Fibroids, no preoperative treatment is needed just hysteroscopic removal. For type 1 Fibroids of less than $3 \mathrm{~cm}$, there are two options either hysteroscopic removal directly or one or two courses of three months UPA followed by hysteroscopic removal if insufficient response to UPA. For type 1 Fibroids more than $3 \mathrm{~cm}$ or Type 2 Fibroids UPA can be given in three months one or two courses. After treatment either Fibroid will reduce in size that no more distort uterine cavity or if still need removal then due to reduction in size hysteroscopic removal is easy. If multiple fibroids or belong to Class 2-5 then 2 courses of three month UPA can be given with following possible outcome:

- $\quad$ Size of fibroid is reduced by more than $50 \%$ and no more distortion of uterine cavity so no further treatment is required and patient can try for natural conception.

- If fibroid size reduced by more than $25 \%$ but less than 50 $\%$ so still need surgery but benefit is that removal will be possible by laparoscopy rather than open surgery.

- If fibroid size reduced by more than $25 \%$ but less than $50 \%$ and still distorted uterine cavity two options should be considered with the patient either prolong medical therapy for another two courses of three months or offer surgical treatment.

- If reduction in size of fibroid not sufficient then surgery is needed. Indication for surgery depends on size and location of fibroid $[1,8,9]$. Premenopausal women presenting with symptomatic fibroids who want to preserve their uterus, similar principle of management as for women less than 40 years of age with type 0 or 1 Fibroids.
Long term intermittent therapy is suggested if type 2 fibroids or multiple large fibroids of Class 2-5. Patient can be observed after 2 courses, if size of fibroid is reduced by more than $50 \%$ or till symptoms reappear. Regarding additional courses further data is needed to determine median time before recurrence. If reduction of fibroid size is not sufficient then there is need for myomectomy $[1,9,10]$. If size of fibroid reduced by more than $25 \%$ but less than $50 \%$ then additional UPA courses may be needed. It is possible to wait until symptoms recur. So available options in such cases are either treatment with UPA, Uterine artery embolization or hysterectomy [2,8-10].

\section{Conclusion}

UPA is found to be effective medical treatment as alternative to surgery, while managing fibroid uterus. So far with studies, UPA is proven to be a good option for fibroid treatment both as preoperatively as well as long term intermittent courses. UPA causes improvement of symptoms associated with Fibroid uterus like pain, bleeding, anaemia as well as reduces size of fibroid also. UPA did not have any effect on ovarian reserve so far but still need more studies to confirm its effect on ovarian function. Endometrial monitoring is needed with long term intermittent courses for UPA treatment. UPA use is associated with reduced need for surgeries. Further studies are needed to assess liver injury following UPA treatment as well as long term outcome of myomectomies after treatment. More data is needed for pregnancies outcome conceived after treatment with UPA in terms of miscarriage, preterm labour, mode of delivery and after delivery complications.

\section{References}

1. Donnez J, Arriagada P, Donnez O, Dolmans MM (2015) Current Management of Myomas: the Place of Medical Therapy with the Advent of Selective Progesterone Receptor Modulators. Current Opinion in Obstetrics and Gynecology 27: 422-431.

2. Safrai M, Chill H, Salzman R, Shushan A (2017) Selective Progesterone Receptor Modulators for the Treatment of Uterine Leiomyomas. Obstetrics \& Gynecology 130: 315-318.

3. Chabbert-Buffet N, Pintiaux-Kairis A, Bouchard P (2007) Effects of the Progesterone Receptor Modulator VA2914 in a Continuous Low Dose on the Hypothalamic-pituitary-Ovarian axis and Endometrium in Normal Women: A Prospective, Randomized Placebo Controlled Trial. Journal of Clinical Endocrinology \& Metabolism 92(9): 3582-3589.

4. Donnez J, Dolmans MM (2016) Uterine Fibroid Management: From the Present to the Future. Human Reproduction Update 22(6): 665-686.

5. Stewart EA, Laughlin Tommaso SK, Catherino WH, Lalitkumar S, Gupta D, et al. (2016) Uterine Fibroids. Nature Reviews Disease Primers.

6. Donnez J, Donnez O, Dolmans MM (2016) Safety of Treatment of Uterine Fibroids with the Selective Progesterone Receptor Modulator, Ulipristal Acetate. Expert Opinion Drug Safety 15(12): 1679-1686.

7. Bestel E, Donnez J (2014) The Potential of Selective Progesterone Receptor Modulators for the Treatment of Uterine Fibroids. Expert Review of Endocrinology \& Metabolism 9: 79-92.

8. Donnez J, Hudecek R, Donnez O, Matule D, Arhendt HJ, et al. (2015) Efficacy and Safety of Repeated Use of Ulipristal Acetate in Uterine Fibroids. Fertil Sertil 103(2): 519-527 e3. 
9. Kalampokas T, Kamath M (2016) Ulipristal Acetate for Uterine Fibroids: A Systematic Review and Meta-analysis. Gynecological Endocrinology 32: 91-99.

ISSN: 2574-1241

DOI: $10.26717 /$ BJSTR.2020.27.004520

Tahira Shahzadi. Biomed J Sci \& Tech Res

(C) This work is licensed under Creative Submission Link: https://biomedres.us/submit-manuscript.php
10. Pérez López FR (2015) Ulipristal Acetate in the Management of Symptomatic Uterine Fibroids: Facts and Pending Issues. Climacteric 18: 177-181.

\begin{tabular}{lll}
\hline & Assets of Publishing with us \\
\hline RESEARCHES & - Global archiving of articles \\
\hline & - Immediate, unrestricted online access \\
\hline
\end{tabular}

